\title{
EDITORIAL
}

\section{Esketamine/ketamine for treatment-resistant depression}

\author{
Acioly L.T. Lacerda ${ }^{1,2}$ iD \\ ${ }^{1}$ Programa de Transtornos Afetivos (PRODAF), Departamento de Psiquiatria, Universidade Federal de São Paulo (UNIFESP), São Paulo, \\ $S P$, Brazil. ${ }^{2}$ Laboratório Interdisciplinar de Neurociências Clínicas (LiNC), UNIFESP, São Paulo, SP, Brazil.
}

Major depressive disorder (MDD) is a disabling, highly prevalent neuropsychiatric disease that affects over $16 \%$ of the world's population. MDD has heterogeneous pathophysiology and multiple potential etiologies. The monoamine hypothesis of depression was developed in the 1950s based on two primary lines of evidence: 1) the effects of reserpine on monoamines; and 2) the findings of different studies on the antidepressant properties of agents with different mechanisms of action, such as monoamine oxidase inhibitors and monoamine reuptake inhibitors, whose common effect was an increase in brain monoaminergic neuronal function. According to this theory, reduced availability of monoamine neurotransmitters in the brain would result in impaired neurotransmission and cognition, which would cause depression. ${ }^{1}$

Continued research has significantly increased our understanding of the disease, but the precise pathophysiology of MDD is still unknown. However, the monoamine hypothesis of depression has "guided" the development of new molecules in the last 5 decades through considerable research efforts toward the development of more efficient and tolerable antidepressants. Different classes of antidepressants all work in slightly different ways and target certain neurotransmitters to modulate mood and behavior. All currently commercially available antidepressants are believed to work by increasing the neurotransmitters serotonin, dopamine or norepinephrine in the synapse.

Despite the availability of over 50 licensed antidepressants, different unmet needs persist in MDD treatment. Response or remission does not occur in a significant proportion of patients. Surprisingly, recent analyses involving 182 controlled trials showed that response rates to antidepressant treatment have not improved in recent decades. $^{2}$ Although unquestionable advances have been made in MDD treatment in recent decades through the development of more tolerable antidepressants, only about one-third of patients attain remission through initial treatment. Even among patients who respond to monoaminergic antidepressants, there is a delayed onset of 4-12 weeks before adequate symptom remission is achieved. Other unmet needs for MDD patients include better tolerability, efficacy for associated symptoms, such as anxiety, sleep and appetite problems, and more effective alternatives for those with treatment-resistant depression

Correspondence: Acioly L. Lacerda, Rua Dr. Pedro de Toledo, 669, $3^{\circ}$ andar, Vila Clementino, CEP 04039-032, São Paulo, SP, Brazil. E-mail: acioly@ institutosinapse.org

Submitted Mar 30 2020, accepted Mar 31 2020, Epub May 112020.
(TRD) and suicide ideation. ${ }^{3}$ Thus, developing MDD treatments with novel mechanisms of action is of utmost importance.

Although the definition of TRD lacks consensus, it generally includes patients who have failed to respond to $\geqslant 2$ antidepressant trials of adequate dose and duration ( $\geqslant 6$ weeks). To manage TRD patients, different evidence-based pharmacological interventions have been proposed, including switching, augmentation and combination strategies. The large $S_{T A R}{ }^{\star} D$ study found modest rates of response and remission for all interventions and have found no differences between augmentation, switching and combination strategies. ${ }^{3}$

Some authors have described the discovery of rapid and robust antidepressant effects of the N-methyl-Daspartate (NMDA) receptor antagonist ketamine as the most important advance in the field of psychiatry in the past half century. Ketamine has demonstrated sustained antidepressant effects in both human and animal models of depression, despite its short half-live (about 3 hours). The antidepressant-like effects of ketamine were first reported in 1975 in animal models, and since the pioneering randomized controlled trial by Berman et al. in $2000,{ }^{4}$ numerous controlled studies have confirmed ketamine's antidepressant effects. In addition, ketamine has been shown to meet different gaps in MDD treatment, including a rapid onset of action, high response rates (up to $85 \%$ ) in TRD, and efficacy in suicidality. More recently, the U.S. Food and Drug Administration (FDA) and the European Medicines Agency (EMA) have approved the use of the enantiomer esketamine, based on results of different phase III randomized controlled trials demonstrating its efficacy in TRD. ${ }^{5}$

The antidepressant action of ketamine/esketamine appears to be related to the modulation of different signaling pathways that have been implicated in the pathophysiology of MDD. Ketamine causes a robust increase in both glutamate and dopamine release in the prefrontal cortex. It also rapidly reverses the effects of chronic stress, such as decreasing the density of dendritic spines and synapse loss in the prefrontal cortex. Improved neuroplasticity in brain regions implicated in depression pathophysiology, such as the hippocampus and prefrontal cortex, has been related to increased protein synthesis

How to cite this article: Lacerda ALT. Esketamine/ketamine for treatment-resistant depression. Braz J Psychiatry. 2020;42:579-580. http://dx.doi.org/10.1590/1516-4446-2020-0996 
associated with the activation of the brain-derived neurotrophic factor receptor tropomyosin receptor kinase B (TrkB), as well as with the deactivation of eukaryotic elongation factor 2 (eEF2). In addition, activation of the mammalian target of rapamycin (mTOR) pathway results in increased expression of synaptic proteins. Moreover, ketamine affects the functioning of numerous cellular effectors of the local inflammatory process very early in its course through direct action on key mechanisms of innate immunity. ${ }^{6}$

This new therapeutic option is much needed for TRD patients, since a large subgroup of patients do not benefit from monoamine antidepressants. Given the few options for TRD patients, the approval of a rapid-acting, highly effective antidepressant is an exciting development. However, different concerns persist about the safety of ketamine/esketamine. Although there is an ongoing longterm, open label study examining the efficacy of the repeated use of esketamine, long-term effects associated with prolonged maintenance treatment are still unknown. In addition, neuroadaptations associated with the development of addiction in preclinical studies have not been ruled out in humans. Finally, no long-term study has evaluated relapse rates after repeated-dose treatment. As with clozapine, the drug reserved for treatment-resistant schizophrenia, the use of ketamine/esketamine should be rigorously monitored.

\section{Acknowledgements}

ALTL has received grants from Conselho Nacional de Desenvolvimento Científico e Tecnológico (CNPq) and
Fundação de Amparo à Pesquisa do Estado de São Paulo (FAPESP).

\section{Disclosure}

ALTL has received grants and personal fees from Janssen Pharmaceutical; has received personal fees from Daiichi Sankyo, Cristalia Produtos Químicos e Farmacêuticos, Libbs, Pfizer, Myralis Farma, Aché Laboratórios, Hypera Pharma, and Sanofi-Aventis; and has received grants from Eli Lilly, $H$. Lundbeck $A / S$, Servier Laboratories, Hoffman-La Roche, and Forum Pharmaceuticals.

\section{References}

1 Jesulola E, Micalos P, Baguley IJ. Understanding the pathophysiology of depression: from monoamines to the neurogenesis hypothesis model - are we there yet? Behav Brain Res. 2018;341:79-90.

2 Papakostas GI, Fava M. Does the probability of receiving placebo influence clinical trial outcome? A meta-regression of double-blind, randomized clinical trials in MDD. Eur Neuropsychopharmacol. 2009; 19:34-40.

3 Rush AJ, Trivedi MH, Wisniewski SR, Nierenberg AA, Stewart JW, Warden $D$, et al. Acute and longer-term outcomes in depressed outpatients requiring one or several treatment steps: a STAR ${ }^{\star} D$ report. Am J Psychiatry. 2006;163:1905-17.

4 Berman RM, Cappiello A, Anand A, Oren DA, Heninger GR, Charney DS, Krystal JH. Antidepressant effects of ketamine in depressed patients. Biol Psychiatry. 2000;47:351-4.

5 Hashimoto K. Rapid-acting antidepressant ketamine, its metabolites and other candidates: a historical overview and future perspective. Psychiatry Clin Neurosci. 2019;73:613-27.

6 Ghasemi M, Phillips C, Fahimi A, McNerney MW, Salehi A. Mechanisms of action and clinical efficacy of NMDA receptor modulators in mood disorders. Neurosci Biobehav Rev. 2017;80:555-72. 